\title{
Tin Ethyl Etiopurpurin
}

National Cancer Institute

\section{Source}

National Cancer Institute. Tin Ethyl Etiopurpurin. NCI Thesaurus. Code C1255.

A synthetic purpurin with photosensitizing activity. T in ethyl etiopurpurin preferentially accumulates in tumor cells due to an increased rate of metabolism. Upon exposure to a light source, this agent absorbs light, forming an extended high energy conformational state that produces high quantum yields of sing let oxygen with local cytotoxic effects.

$(\mathrm{NCl04})$ 